\title{
SUZAKU OBSERVATION OF THE BLACK HOLE CANDIDATE MAXI J1836-194 IN A HARD/INTERMEDIATE SPECTRAL STATE
}

\author{
R. C. Reis ${ }^{1}$, J. M. Miller ${ }^{1}$, M. T. Reynolds ${ }^{1}$, A. C. Fabian ${ }^{2}$, and D. J. Walton ${ }^{2}$ \\ ${ }^{1}$ Department of Astronomy, University of Michigan, Ann Arbor, Michigan, MI 48109, USA \\ 2 Institute of Astronomy, University of Cambridge, Madingley Road, Cambridge CB3 OHA, UK \\ Received 2011 November 23; accepted 2012 March 14; published 2012 May 2
}

\begin{abstract}
We report on a Suzaku observation of the newly discovered X-ray binary MAXI J1836-194. The source is found to be in the hard/intermediate spectral state and displays a clear and strong relativistically broadened iron emission line. We fit the spectra with a variety of phenomenological, as well as physically motivated disk reflection models, and find that the breadth and strength of the iron line are always characteristic of emission within a few gravitational radii around a black hole. This result is independent of the continuum used and strongly points toward the central object in MAXI J1836-194 being a stellar mass black hole rotating with a spin of $a=0.88 \pm 0.03$ (90\% confidence). We discuss this result in the context of spectral state definitions, physical changes (or lack thereof) in the accretion disk, and on the potential importance of the accretion disk corona in state transitions.
\end{abstract}

Key words: accretion, accretion disks - black hole physics - line: profiles - relativistic processes - X-rays: binaries - X-ray: individual (MAXI J1836-194)

Online-only material: color figures

\section{INTRODUCTION}

The X-ray spectra of X-ray binaries provide important clues on the nature of the compact objects and on the broad properties of the accretion flow. In particular, the various reflection features endemic to stellar mass black hole binaries in all active states have been successfully used to constrain the dimensionless spin parameter of various black holes in binary systems, as well as providing invaluable insight into the manner in which accretion flow varies with mass accretion rate (e.g., Miller 2007). By far the most prominent - and probably the most importan$\mathrm{t}-\mathrm{of}$ these reflection features is the relativistic iron line appearing at approximately $6.4-6.97 \mathrm{keV}$ depending to the ionization state of the emitting material (e.g., Tanaka et al. 1995). Suzaku combines exceptional energy resolution below $\approx 10 \mathrm{keV}$ with broadband observation, and, as such, is unique among other current X-ray satellites in the study of black hole transients.

The observed spectrum often exhibits the presence of thermal emission, originating in an optically thick accretion disk together with a hard component often referred to as the corona, and reflection features. There is a complex link between the disk, coronal hard X-rays, and reflection emissions (e.g., Done et al. 2007), which manifests in various spectral states (see Remillard \& McClintock 2006 and Belloni 2010). Characterizing the driving force between these state transitions is a fundamental challenge for both theoretical and observational studies of accretion-flow properties.

The prevailing paradigm requires that in quiescence (very low $\dot{m}$ ), the inner accretion disk is fully replaced by an advectiondominated accretion flow (ADAF; e.g., Esin et al. 1997). This has led to the idea that the transition between active states is a manifestation of changes in the innermost extent of the accretion disk, so that a transition from the disk-dominated high/soft state to a power-law-dominated low/hard state marks the point of the disk recession. The constant presence of radio jets in the low/hard state can also be associated, albeit in a qualitative manner, with the truncation radius. Thus, it is clear that knowledge of the inner extent of the accretion disk can have fundamental consequences to our understanding of the nature of the accretion flow at low $\dot{m}$ as well as the connection between accretion disk, corona, and radio jets. This radius can be determined via the study of both the continuum emission from the accretion disk or by the reflected iron emission line, again making Suzaku, with its broadband coverage and high spectral resolution, ideal for this science. In fact, the advent of $X M M-N e w t o n$ and Suzaku has strongly challenged the paradigm that the accretion disk is truncated in the bright phases of the low/hard state in black hole binaries (but see Done \& Diaz Trigo 2010; also see Miller et al. 2010).

This challenge is exemplified by the recent Suzaku and XMM-Newton observations of XTE J1752-223 (Reis et al. 2011) and the $42 \mathrm{ks}$ XMM-Newton observation of XTE J1652-453 (Hiemstra et al. 2011). XTE J1752-223 was caught during the decay of its 2009 outburst in both the intermediate (Suzaku) and low/hard (XMM-Newton) spectral states. Interestingly, in both observations we found the presence of a strong, relativistic iron emission line which independently yielded strong constraints on the inner radius: $R_{\text {inner }}=2.8_{-0.1}^{+0.3} r_{\mathrm{g}}$ and $3.9 \pm 0.5 r_{\mathrm{g}}$ (90\% confidence; Reis et al. 2011) for the intermediate and low/hard state, respectively, as well as thermal disk components clearly following the $L \propto T^{4}$ relation expected for geometrically thin accretion disk, thus strongly ruling out disk truncation in either state. Similar results have been found for XTE J1652-453, where Hiemstra et al. (2011) find the disk to be at $\approx 4 r_{\mathrm{g}}$ in the hard/intermediate state, and for GX 339-4 where the disk does not appear to truncate until at least $10^{-3} L_{\text {Edd }}$ (Miller et al. 2006; Reis et al. 2008; Tomsick et al. 2009; Wilkinson \& Uttley 2009).

In order to determine whether these sources are anomalous, or if state transitions are really not linked with a recession of the innermost extent of an optically thick, geometrically thin accretion disk, we urgently need observations at low fractions of the Eddington limit, down to $10^{-4}$ and below. However, it is clear that at in some phases of the low/hard state, at least as defined by McClintock et al. (2006), the disk does not appear to truncate beyond the radius of the Innermost Stable Circular 
Orbit (ISCO). Whether this is due to a different phase of the low/hard state-i.e., an "ADAF state"-or whether the disk only truly begin to truncate at much lower $\dot{m}$ remains to be seen. Either way, it is clear that the disk plays an important role in state transitions and even jet creation, be it by physically truncating and allowing for the existence of an inner ADAF zone or by dissipating less gravitational energy and allowing for a more powerful accretion disk corona.

The shape of the iron line is determined by the relative depth of the disk within the potential well of the black hole and, as such, conveys information on its spin. Understanding the role of black hole spin $\left(a=c J / G M^{2},-1 \leqslant a \leqslant 1\right)$ in shaping accretion flows onto and jets from black holes is an important goal, having strong repercussion in all areas of astronomy. Stellar mass black holes, for example, are likely to gain most of their angular momentum during birth, and their spin is a consequence of the supernovae that results in the creation of the central black hole (see e.g., Miller et al. 2011). Knowing the spin distribution for these objects thus provides a window into the nature of one of the most powerful explosions in the universe. At present, we have approximately a dozen spin measurements made by the use of the relativistic iron lines (e.g., Miller et al. 2008, 2009; Reis et al. 2008, 2009a, 2011) and a handful obtained from the thermal disk continuum (McClintock et al. 2006; Gou et al. 2009; Steiner et al. 2011). However, in order to make any claim on the possible role of spin on, for example, radio jet power (Fender et al. 2010), we need to increase our spin demographics.

In this paper, we draw on the recent Suzaku TOO observations of the nearly discovered black hole candidate MAXI J1836-194 to learn about the nature of the innermost accretion flow in this source, and to increase our black hole spin demographics. The following section summarizes all the observations of the source and details the current Suzaku observation. Section 3 begins by exploring some of the more phenomenological models used to explain the spectra of X-ray binaries and confirms the black hole nature of the central source. We conclude this section by using a fully self-consistent and physically motivated model to estimate the spin parameter of the black hole in MAXI J1836-194. Section 4 summaries our results and discusses the implications for current ideas of black hole state transitions and interpretations.

\section{OBSERVATION AND DATA REDUCTION}

MAXI J1836-194 was discovered by the MAXI/GSC observatory on 2011 August 30 (Negoro et al. 2011). Its current evolution, at various wavelengths, have been reported in various ATels (Kennea et al. 2011; Cenko et al. 2011; Strohmayer \& Smith 2011; Rau et al. 2011; Nakahira et al. 2011; Del Santo et al. 2011; Trushkin et al. 2011; Russell et al. 2011) with the latest report by Russell et al. (2011) strongly suggesting that MAXI J1836-194 is indeed a black hole X-ray binary based on Very Large Telescope mid-IR detections. Similar conclusions were made by Miller-Jones et al. (2011) based on EVLA radio detections. The $6 \mathrm{ks} R X T E /$ Proportional Counter Array observation of MAXI J1836-194 reported by Strohmayer \& Smith (2011) had the spectrum described by an absorbed power law with photon index of 1.84 . The authors reported the presence of an iron emission line at $6.3 \pm 0.2 \mathrm{keV}$ together with a smeared edge at $7.2 \pm 0.2 \mathrm{keV}$ and alluded to a reflection interpretation of these features. The quoted $3-20 \mathrm{keV}$ flux of $9.8 \times 10^{-10} \mathrm{erg} \mathrm{cm}^{-2} \mathrm{~s}^{-1}$ is remarkably similar to that of

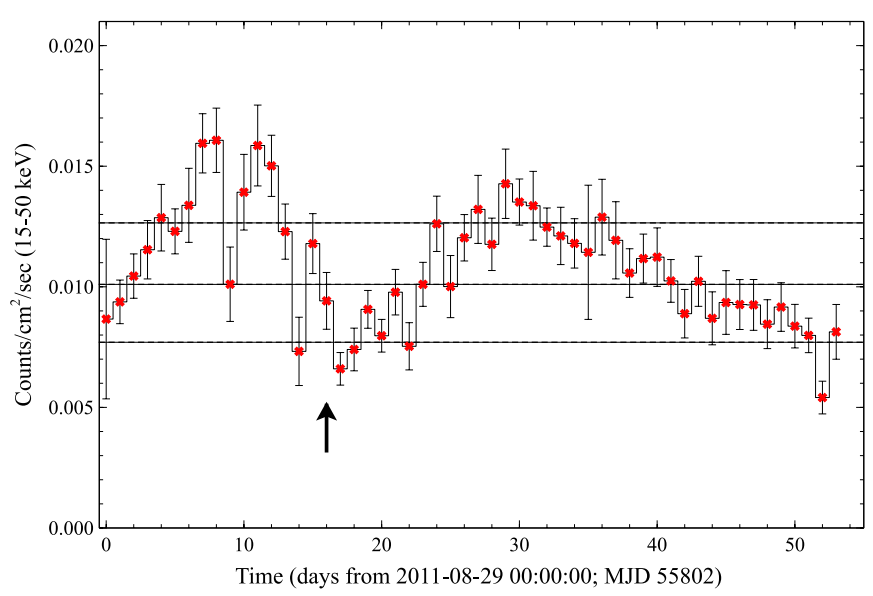

Figure 1. Swift/BAT light curve for MAXI J1836-194 in the 15-50 keV energy range. The dashed horizontal lines show the average rate and $\pm 1 \sigma$ standard deviation for this source since its discovery. The arrow shows the time of the Suzaku observation reported in this paper. Light curve is available at http://heasarc.gsfc.nasa.gov/docs/swift/results/transients/weak/ MAXIJ1836-194/.

(A color version of this figure is available in the online journal.)

XTE J1752-223 in a similar state $\left(\approx 11 \times 10^{-10} \mathrm{erg} \mathrm{cm}^{-2} \mathrm{~s}^{-1}\right.$; $\Gamma=1.83 \pm 0.02$; Reis et al. 2011).

Figure 1 shows the hard X-ray evolution of MAXI J1836-194 as observed by the Swift/BAT Hard X-ray Transient Monitor provided by the Swift/BAT team. Suzaku observed the source on 2011 September 14 for a total of $10.7 \mathrm{ks}$ during a period where the 15-50 keV flux showed a slight decrease. The three operating detectors constituting the X-ray Imaging Spectrometer (XIS; Koyama et al. 2007) on board of Suzaku were operated in the $1 / 4$ window "burst" mode with both front- and backilluminated detectors in the $3 \times 3$ and $5 \times 5$ editing modes. Using the latest HEASOFT v6.11.1 software package we processed the unfiltered event files for each CCD following the Suzaku Data Reduction Guide. ${ }^{3}$ Due to the observation having been preformed in "burst" mode, we started by producing detailed good time intervals (GTIs) using the FTOOL XISTIME and setting the option to "bstgti=yes." New attitude files were then created using the AEATTCOR script ${ }^{4}$ (Uchiyama et al. 2008) in order to correct for shift in the mean position of the source caused by the wobbling of the optical axis. The FTOOL XISCOORD was used to create new event files which were then further corrected by re-running the Suzaku pipeline with the latest calibration, as well as the associated screening criteria files. The GTIs provided by the XIS team were also employed in all cases to exclude any possible telemetry saturations. XSELECT was used to extract spectral products from these event files.

In order to estimate the level of pile up suffered by the data we used the script PILE_EST ${ }^{5}$ (Davis 2001) to create a pileup map out of a Suzaku event data file. After experimenting with various extraction regions we choose to employ a box annulus region with a width of 240 pixels $\left(\sim 250^{\prime \prime}\right)$ and a height of 290 pixels $\left(\sim 302^{\prime \prime}\right)$, and an inner radius of 70 pixels $\left(\sim 73^{\prime \prime}\right)$. This resulted in a maximum pileup fraction of $2 \%$. Background spectra were extracted from a circular region having a radius of $100^{\prime \prime}$ elsewhere on the same chip. Individual ancillary response files (arfs) and redistribution matrix files (rmfs) were produced

\footnotetext{
3 http://heasarc.gsfc.nasa.gov/docs/suzaku/analysis/

4 http://space.mit.edu/cxc/software/suzaku/aeatt.html

5 http://space.mit.edu/ASC/software/suzaku/pest.html
} 
with the script XISRESP ${ }^{6}$ — which calls the tools XISRMFGEN and XISARFGEN_-with the "medium" input.

Finally, we combined the spectra and response files from the two front-illuminated instruments (XIS0 and XIS3) using the FTOOL ADDASCASPEC to increase signal to noise. The FTOOL GRPPHA was used to give at least 100 counts per spectral bin in a total of 512 energy channels. The nominal energy range covered by the XIS detectors is from $\sim 0.2$ to $12 \mathrm{keV}$. However, there are still calibration issues below $\sim 1 \mathrm{keV}$, which are especially severe in the burst clocking mode. Therefore, we do not consider data below $1.2 \mathrm{keV}$ or above $10 \mathrm{keV}$, following the analyses of XTE J1752-223 presented by Nakahira et al. (2012). The energy band of 1.6-2.4 keV is also excluded to avoid large systematics uncertainties in the effective area near the silicon $\mathrm{K}$ and gold $\mathrm{M}$ edge. The smaller effective area, together with the fact that the out-of-time event rate is more significant in the BI instrument, compared to the FI, means that at the energies considered here (i.e., above $1.2 \mathrm{keV}$ ) the BI data contain even larger uncertainties, and for this reason we did not use XIS1 data in this paper.

We processed the Hard X-ray Detector (HXD; Takahashi et al. 2007) with the standard criteria. The appropriate response file (ae_hxd_pinxinome11_20110601.rsp) for XIS-nominal pointing was downloaded ${ }^{7}$ and the data were reprocessed in accordance with the Suzaku Data Reduction Guide. As the nonX-ray background (NXB) file was yet to be created by the HXD team at the time of writing, we estimate the NXB by extracting the earth-occulted data" $(\mathrm{ELV}<-5) .{ }^{8}$ Dead time corrections were applied with HXDDTCOR. The contribution from the cosmic X-ray background (CXB) was simulated using the form of Boldt (1987), with appropriate normalization for the XIS nominal pointing, resulting in a CXB rate of 0.019 count $^{-1}$. The earth-occulted NXB and CXB spectra were then combined using MATHPHA to give a total background spectrum, to which a $2 \%$ systematic uncertainty was added. The source spectrum was finally grouped to at least 100 counts per spectral bin. The PIN spectrum is restricted to the $15.0-42.0 \mathrm{keV}$ energy range and fits simultaneously with the XIS data by adding a normalization factor, which is set to 1.16 in all fits with respect to that of the FI spectrum as recommenced by the Suzaku data analysis guide. To test the robustness of our result, in the following sections we also investigate the effect of allowing this cross-normalization to vary. All errors reported in this work are $90 \%$ confidence errors obtained by allowing all parameters to vary, unless otherwise noted.

\section{DATA ANALYSES AND RESULTS}

\subsection{Exploring Phenomenological Models}

In order to compare the spectral properties of MAXI J1836-194 with past work on other X-ray binaries, we begin by fitting the data with a simple combination of an absorbed power law together with a DISKBB (Mitsuda et al. 1984) model. Figure 2 shows this fit with the $4-7 \mathrm{keV}$ range ignored in order to best model the continuum. The total $0.01-100 \mathrm{keV}$ unabsorbed flux is $\sim 7.8 \times 10^{-9} \mathrm{erg} \mathrm{cm}^{-2} \mathrm{~s}^{-1}$ of which approximately

\footnotetext{
6 http://suzaku.gsfc.nasa.gov/docs/suzaku/analysis/xisresp

7 http://www.astro.isas.ac.jp/suzaku/analysis/hxd/

8 This has now become available and we have checked the consistency in the results. The "tuned" background has a $15-45 \mathrm{keV}$ flux that is approximately $3 \%$ less than the earth-occulted background and is fully consistency within errors. The spectral shape is also fully consistent with one another and does not alter the results presented here.
}

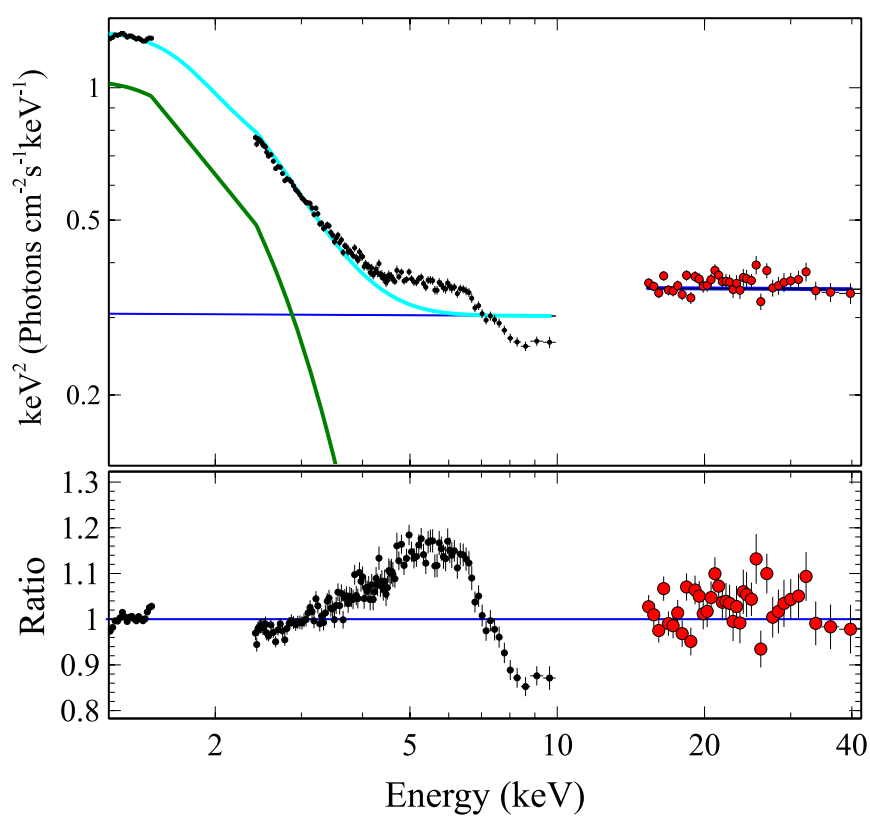

Figure 2. Response unfolded $v F v$ spectrum of MAXI J1836-194. The total, disk, and power-law components are shown as cyan, blue, and green solid lines, respectively. Bottom: data model ratio to an absorbed DISKBB plus POWERLAW fit ignoring the $4-7 \mathrm{keV}$ energy range.

(A color version of this figure is available in the online journal.)

$42 \%$ is associated with the accretion disk. For comparison with the standard work of McClintock et al. (2006) and Belloni (2010), we quote the total $2-20 \mathrm{keV}$ unabsorbed flux as $\sim 1.5 \times 10^{-9} \mathrm{erg} \mathrm{cm}^{-2} \mathrm{~s}^{-1}$ and a disk fraction of $\approx 0.26$. Combined with a spectral index of $\sim 2.0$, this observation of MAXI J1836-194 is consistent with having caught the source in the hard/intermediate spectral state. A further hint of this is also seen in Figure 1, where it is clear that the source was observed during a time of slight decrease in the hard X-ray flux and suggests a short excursion away from the low/hard state.

In Figure 2, the 4-7 keV energy range is shown above the continuum in order to highlight the presence of various features in this range. A possible explanation used by a number of authors to account for the residuals seen in Figure 2 assumes that these features are a combination of a narrow iron $\mathrm{K} \alpha$ emission line and its associated absorption edge, which, when arising from the region around a black hole, suffers from a high degree of smearing and thus is better described by the SMEDGE model (e.g., Ebisawa et al. 1994). A fit with such a smeared edge having an energy of $7.11 \mathrm{keV}$ (as expected from neutral iron) and a width of $10 \mathrm{keV}$ was not able to account for the residuals $\left(\chi^{2} / v=630.9 / 328\right.$ Model 1a; Table 1 and Figure 3$)$, with broad residuals remaining both above and below the edge energy. Adding a narrow $(\sigma=1 \mathrm{eV})$ Gaussian line at $6.4 \mathrm{keV}$ did not improve the residuals in any way. A much better fit is indeed achieved when the energies of both the Gaussian line and that of the smeared edge are allowed to be free and the emission line is allowed to be broad $\left(\chi^{2} / v=380.3 / 324\right.$; Model $1 \mathrm{~b}$; Table 1). The neutral hydrogen density found in these models is also mildly consistent with the value of $2.0 \pm 0.4 \times 10^{21} \mathrm{~cm}^{-2}$ presented by Kennea et al. (2011) based on a Swift/XRT observation. We will discuss possible reasons for the variation in $N_{\mathrm{H}}$, as observed between the different models, in Section 4. The scenario so far presented here, however, has an edge energy of $\sim 5.4 \mathrm{keV}$ which is much less than the value for neutral iron absorption; itself a lower limit since iron is likely to be highly 
Table 1

Phenomenological Fits Used to Determine the Robustness of the Emission Line

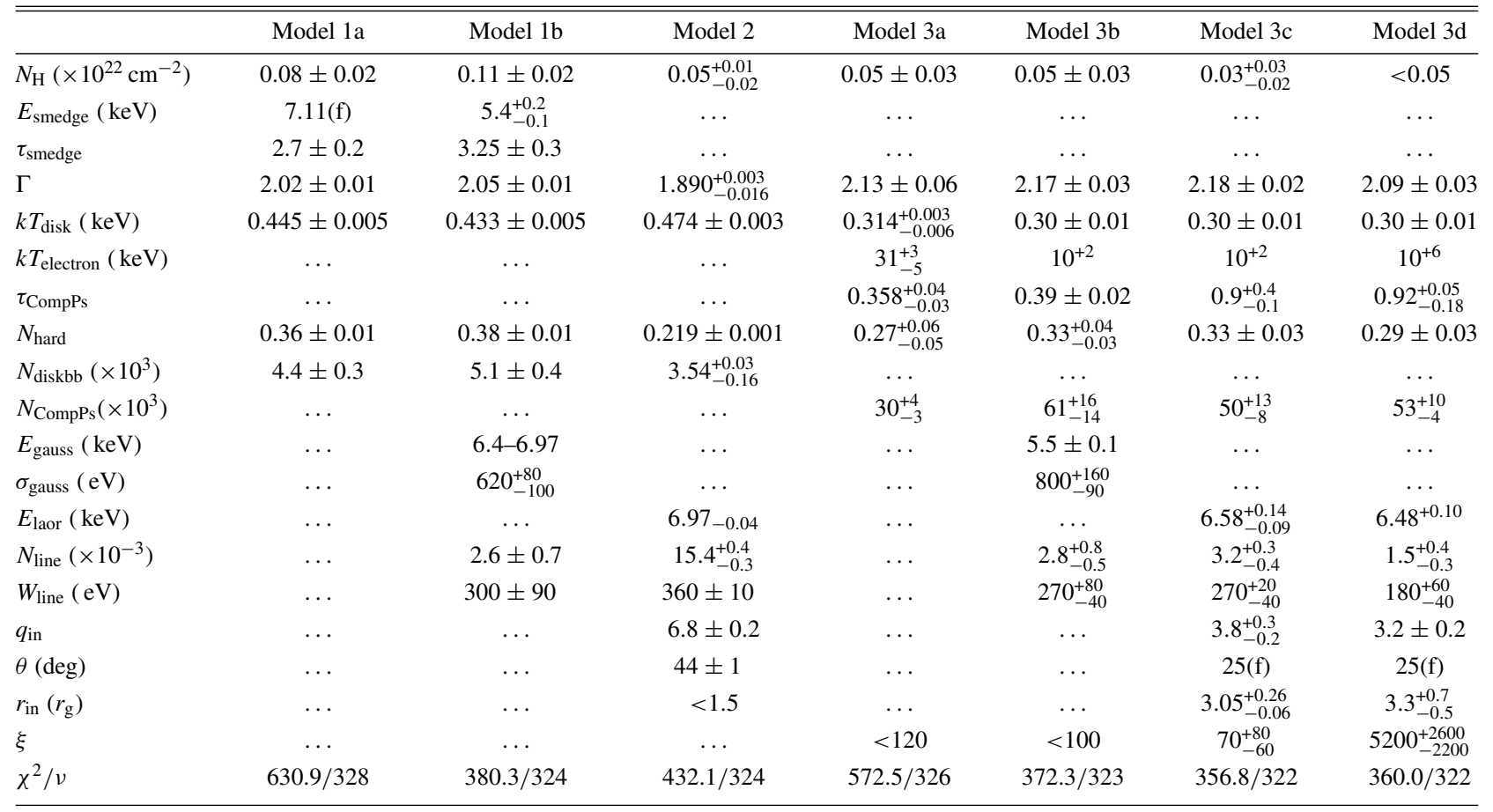

Notes. Results of phenomenological fits with a variety of continuum models. The continuum in Model 1 is assumed to consist of a simple power law having a normalization $N_{\text {hard }}$ and a DISKBB component. The feature around the 6-7 keV range is modeled with a smeared edge (Model 1a) together with a further Gaussian line (Model 1b). Model 2 replaces the smeared edge and the Gaussian line with a single relativistic line component. Model 3 replaces the DISKBB and power-law models with the Comptonization code of Poutanen \& Svensson (1996) and the model PEXRIV of Magdziarz \& Zdziarski (1995), respectively. For Model 3, a "slab" geometry was assumed and the reflection option in compPS was deactivated. A lower limit for the electron temperature of $10 \mathrm{keV}$ was imposed. The feature is modeled with a broad Gaussian (Model 3b) and a relativistic line (Model 3c). Model $3 \mathrm{~d}$ self-consistently convolves the PEXRIV model with the same parameters as the relativistic line since they both originate from the same region. All errors are $90 \%$ confidence.

ionized in the inner parts of the accretion disk. In fact, it is more likely that the edge is trying to compensate for a broad emission feature (see Section 4) and, for this reason, although the model is detailed in Table 1, we do not believe it to convey any physical information.

Given the obvious presence of a broad line feature in Figure 2, we replaced the SMEDGE model with an LAOR line profile (Laor 1991) as expected if emission is coming from the inner disk around a black hole. The line energy is constrained to lie between 6.4 and $6.97 \mathrm{keV}$, thus encompassing the full range of possible ionization states of iron. We start in Model 2 with a simple power-law emissivity profile such that $\epsilon(r) \propto r^{-q_{\text {in }}}$. The outer disk radius was frozen at the maximum value in the model of $400 r_{\mathrm{g}}$. This model is detailed in Table 1 and resulted in a satisfactory fit with $\left(\chi^{2} / v=432.1 / 324\right)$. Allowing for a broken power-law emissivity profile with indices $q_{\text {in }}$ within a radius $r_{\text {break }}$ and $q_{\text {out }}$ beyond further improved the fit $\left(\Delta \chi^{2}=13.5\right.$ for 2 degrees of freedom). This fit has an emissivity index of $q_{\text {in }} \sim 7$ within a radius of $\sim 6 r_{\mathrm{g}}$ and then breaks to $q_{\text {out }} \sim 2.6$. In both of these instances, the inner radius obtained is very low implying that not only is the central object in MAXI J1836-194 a stellar mass black hole, it is also likely to be rapidly spinning. In all models considered so far, the DISKBB component consistently required a disk with a temperature of approximately $0.45 \mathrm{keV}$.

It has been suggested by a number of authors that this simple view of a multicolor disk is not appropriate and should be replaced with a broader disk model. Kolehmainen et al. (2011) further suggested that the broad iron line constantly seen in a number of stellar mass black hole binaries might be an artificial effect caused by the usage of narrower disk components similar to DISKBB used here. Despite the noticeable problems with this interpretation-iron lines are mostly seen in black hole binaries in the low/hard state where the disk temperature is relatively cold and its contribution to the spectrum at $\approx 6 \mathrm{keV}$ is unimportant-we nonetheless test the effect this may have on the residuals seen between 4 and $7 \mathrm{keV}$ (Figure 2), by replacing both the DISKBB, LAOR, and POWERLAW components with compPS (Poutanen \& Svensson 1996) and PEXRIV (Magdziarz \& Zdziarski 1995). The former fully characterizes the process of Comptonization for a variety of coronal geometries, electron distributions, and seed photons injection geometries. We used the code assuming a purely thermal electron distribution in the corona $(\mathrm{Gmin}=-1)$. The PEXRIV model represents a exponentially cutoff power-law spectrum ${ }^{9}$ reflected from ionized material. When the reflection fraction, $R>0$, this model gives the sum of the illuminating power-law hitting the ionized disk together with the corresponding reflection component (Fe-edge and Compton hump) but it does not include the $\mathrm{Fe}$ $K \alpha$ emission line. We initially have the ionization parameter ( $\xi=4 \pi F / n \mathrm{erg} \mathrm{cm} \mathrm{s}^{-1}$, where $F$ is the incident flux and $n$ is the number density of hydrogen nuclei), reflection fraction, and inclination of the PEXRIV component frozen at $1000 \mathrm{erg} \mathrm{cm} \mathrm{s}^{-1}$, $1^{\circ}$ and $25^{\circ}$, respectively, in line with the values used for XTE

\footnotetext{
9 The cutoff energy is frozen at $300 \mathrm{keV}$, similar to the value used in the reflection model REFLIONX described in detail in the next section.
} 


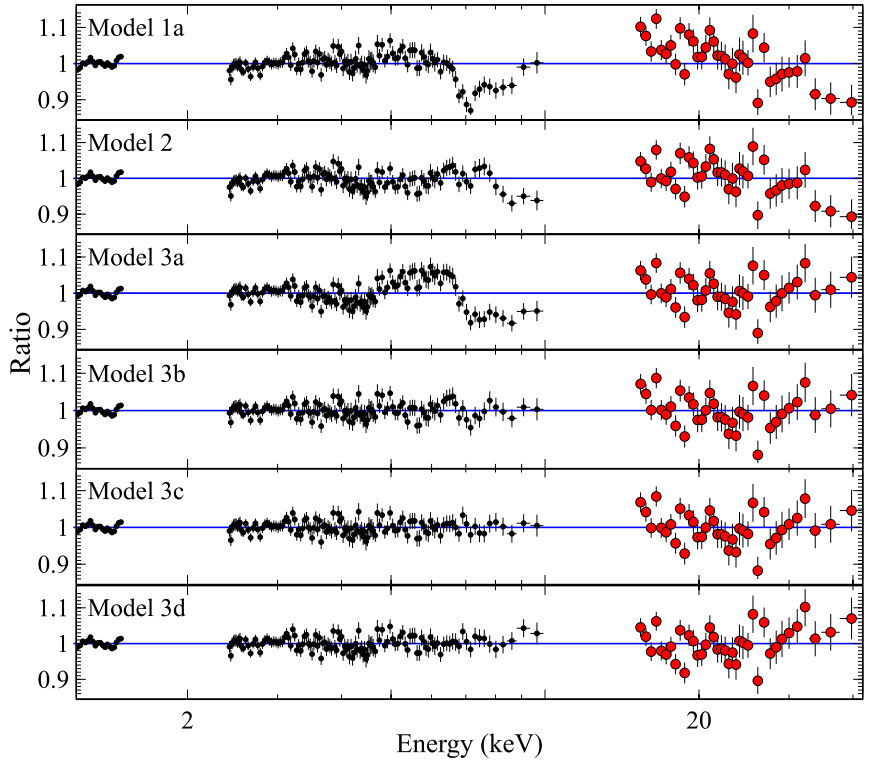

Figure 3. Data/model ratio for the various phenomenological models described in Table 1. From top to bottom: phabs $*$ smedge $*($ diskbb+powerlaw $)$ with the smeared edge energy frozen at $7.11 \mathrm{keV}$; phabs $*($ laor + diskbb+ powerlaw); phabs $*($ compps + pexriv $) ;$ phabs $*($ compps + pexriv + gaussian $) ;$ phabs $*$ (compps + pexriv + laor $) ;$ phabs $*($ compps + kdblur $\otimes$ pexriv + laor $)$.

(A color version of this figure is available in the online journal.)

J1752-223 by Nakahira et al. (2012). The geometry of compPS is chosen to be a "slab" (geom=1) and the covering fraction frozen at 1 . Reflection from the compPS component is turned off (rel_refl=0).

This combination resulted in an unsatisfactory fit with $\chi^{2} / v=629.4 / 327$. Allowing the ionization of PEXRIV to vary did not resolve the problem $\left(\chi^{2} / v=572.5 / 326\right)$, with a broad feature clearly present in the residuals between 4 and $7 \mathrm{keV}$ (Model 3a in Table 1; Figure 2). Further allowing the reflection fraction to change again did not provide a satisfactory fit $\left(\chi^{2} / v=546.8 / 325\right)$. The reflection fraction artificially shoots up to $\sim 2.5$ as the PEXRIV model tries to compensate for the lack of an Fe-emission line by increasing the depth of the iron-edge (see Figure 9). Indeed, adding a broad Gaussian line (Model 3b) resulted in a dramatic improvement, with $\Delta \chi^{2}=$ 200.2 for 3 degrees of freedom compared to Model 3a. However, a line energy of $E_{\text {Gauss }}=5.5 \pm 0.1 \mathrm{keV}$ is not consistent with emission of iron. Furthermore, a width of $\sim 800 \mathrm{eV}$ is highly suggestive of emission from close to the black hole where gravitational broadening effects are important, again taking us back to the need of a relativistic iron line.

We therefore proceed by replacing the Gaussian line in Model $3 \mathrm{~b}$ with the relativistic line expected around a spinning black hole. The inclination in the LAOR model is frozen at $25^{\circ}$, as with PEXRIV. This model immediately improved the quality of the fit $\left(\Delta \chi^{2}=15.5\right.$ for 1 degree of freedom) and, more importantly, brought the emission line energy to a range consistent with emission from iron $\left(E_{\mathrm{Laor}} \approx 6.6 \mathrm{keV}\right)$. The inner radius as obtained from the iron line profile in Model $3 \mathrm{c}$ is again consistent with the central object in MAXI J1836-194 being a rotating black hole. Allowing the reflection fraction in the PEXRIV model to be free yields $R=0.9 \pm 0.2$, and does not change any of the other fit parameters nor does it affect the quality of the fit $\left(\chi^{2} / v=356.4 / 321\right)$. Importantly, Model $3 \mathrm{c}$ as it stand is not physically consistent. If the $\mathrm{Fe}$ line is being emitted in the inner parts of the accretion disk, as appears to be the case, then the other reflection features should also experience the effect of strong gravity. For this reason we convolve the PEXRIV model-which models the illuminating continuum as well as the absorption edge of iron and the Compton reflection hump-with the relativistic kernel KDBLUR (Laor 1991). We force the parameters of KDBLUR to be the same as that of the relativistic iron line. This model (Model 3d) results in a slightly worst quality of fit $\Delta \chi^{2}=3.2$ for the same number of degrees of freedom as the previous model; however, it is now physically consistent. Again, allowing the reflection fraction in the PEXRIV model to be free resulted in an improvement of $\Delta \chi^{2}=4.4$ for 1 degree of freedom, giving $R<0.91$, but did not change the line profile in any way, with the inner radius and emissivity index remaining at $r_{\text {in }}=3.3_{-0.4}^{+0.7} r_{\mathrm{g}}$ and $q_{\text {in }}=3.4 \pm 0.2$, respectively. We also investigated the robustness of the fit with respect with the cross-normalization constant between the XIS and PIN data which we have so far fixed at 1.16. Allowing this to go free barely improved the quality of the fit $\left(\Delta \chi^{2}=1.4\right.$ for 1 degree of freedom) and recovered a value of $1.14_{-0.08}^{+0.03}$, fully consistent with the expected value.

To expedite the computational time, in all incarnations of Model 3 we have assumed that the inner disk inclination has a value of $25^{\circ}$. As a last step in our exploration of these phenomenological models, we allow the inclination to be free and investigate any possible degeneracy this might have on the inner accretion radius and emissivity index. Figure 4 shows the $68 \%, 90 \%$, and $95 \%$ confidence range for both parameters as a function of inclination as well as the inclination as a function of emissivity index. From Figure 4, it can be seen that despite the fact that the inclination is not very well constrained, ranging from anywhere between $5^{\circ}$ and $40^{\circ}$ at the $90 \%$ confidence level, the inner radius of the accretion disk, as obtained solely from the breadth of the line profile using purely phenomenological models, is still consistent with being at or close to the radius of the Innermost Stable Circular Orbit around a rotating black hole. The global minimum, which is marked with a black cross in all panels, still requires $r_{\text {in }}<5.6 r_{\mathrm{g}}$ at the $90 \%$ level; however, we do see the presence of a further solution having a radius which is marginally consistent with the ISCO of a non-rotating black hole together with a very high emissivity index. In Fabian et al. (2012), we showed that a single power-law emissivity profile has only limited validity and that a slope of $\sim 3$ is a fair approximation for an inner disk starting at $r_{\text {in }} \sim 2 r_{\mathrm{g}}$. However, it severely underestimates the profile within $r_{\text {in }} \sim 2 r_{\mathrm{g}}$ and is therefore a poor probe of the innermost region around a rapidly spinning black hole. In that paper, we argued that if $q=3$ is used, then it will likely yield an upper limit to the inner radius and thus a lower limit on the spin if the source is indeed spinning rapidly. The evidence so far points toward the black hole in MAXI J1836-194 not being rapidly spinning, and therefore, from theoretical arguments we should expect an emissivity index close to 3 , similar to the value found in the global minima having an inner radius within $r_{\text {in }}<5.6 r_{\mathrm{g}}$. In the following section, we will investigate these possible degeneracies fully using a number of self-consistent reflection models.

In this section, we have established beyond any reasonable doubt the presence of a strong and mildly broad emission line associated with iron in the Suzaku spectra of MAXI J1836-194. We have shown that different continuum models as well as phenomenological features (such as the blurred edge component) do not eliminate the need for a broad emission line. Our efforts to "remove" the need for a broad emission line are 

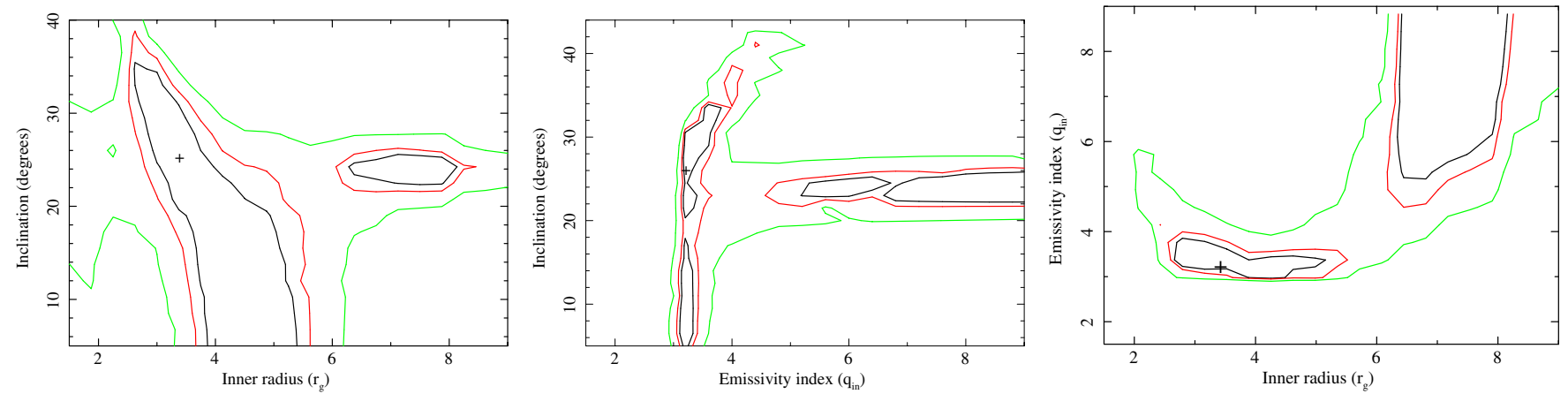

Figure 4. Contour plots investigating the effect of the inclination (left) and emissivity index (right) on the inner radius for Model $3 \mathrm{~d}$. The center panel shows the contour plot for the inclination vs. emissivity index. The $68 \%, 90 \%$, and $95 \%$ confidence range for two parameters of interest are shown in black, red, and green, respectively. The cross marks the global minima. It is clear that even for the most curved continuum where the emission feature would appear the narrowest (i.e., Model 3), the inner extent of the accretion disk, as obtained solely from the phenomenological fit to the iron line profile, is still consistent with not being truncated far beyond the innermost stable circular orbit for a Schwarzschild black hole $\left(6 r_{\mathrm{g}}\right)$ in the hard/intermediate spectral state observed here. However, due to the local minima in these phenomenological models we cannot rule out a disk that is mildly truncated at this stage.

(A color version of this figure is available in the online journal.)

somewhat artificial since reflection is a natural consequence of a system where hard X-rays are impinging on a cold accretion disk. However, it is only by doing so that we can safely rule out any continuum effect on the breadth of the iron emission line. In the following section, we endeavor to interpret the spectra in a fully consistent and physical manner.

\subsection{Physically Self-consistent Modeling}

In all our previous fits, a broad feature has been shown to be robustly present above a thermal disk and power-law-like continuum, peaking around the energies expected for neutral $\mathrm{Fe}$ I $\mathrm{K} \alpha$ emission $(\sim 6.4 \mathrm{keV})$ and highly ionized H-like Fe xxvi Ly $\alpha$ $(\sim 6.97 \mathrm{keV}$; see Figure 2). The natural explanation for this feature is that it is indeed associated with the reprocessing of hard $\mathrm{X}$-ray emission by an accretion disk and, as such, the broad feature observed is the signature of iron fluorescence that has been relativistic broadened due to the strong gravity around the central black hole. Similar reflection features are observed in a wide range of objects ranging from neutron stars (Bhattacharyya \& Strohmayer 2007; Cackett et al. 2008, 2009, 2010; di Salvo et al. 2009; Reis et al. 2009b), stellar mass black holes (Miller 2007; Blum et al. 2009; Reis et al. 2009a; Hiemstra et al. 2011; Walton et al. 2012), and active galactic nuclei (AGNs; Tanaka et al. 1995; Fabian et al. 2009; Miniutti et al. 2009; Schmoll et al. 2009; Walton et al. 2010; Nardini et al. 2011; Brenneman et al. 2011; Reis et al. 2012).

Due to the immense diagnostic potential of reflection features, a large theoretical effort has been devoted to fully characterizing the reflection spectrum expected to arise from such systems (Lightman \& White 1988; George \& Fabian 1991; Matt et al. 1991; Ross \& Fabian 1993; Zycki et al. 1994; Nayakshin et al. 2000; Ballantyne et al. 2001; Ross \& Fabian 2005, 2007; García \& Kallman 2010; García et al. 2011). Among these, the most widely used reflection model is the REFLIONX code of Ross \& Fabian (2005). This model self-consistently calculates the reflection arising from all energetically important ionization states and transitions expected in disks around black holes. At low ionization parameters it reproduces the reflection continuum first described by Lightman \& White (1988), as well as selfconsistently calculating the fluorescent lines; at higher $\xi$, lower$Z$ element becomes ionized which results in a softening of the reflection spectrum. We start by using a combination of REFLIONX, relativistically convolved with KDBLUR, together

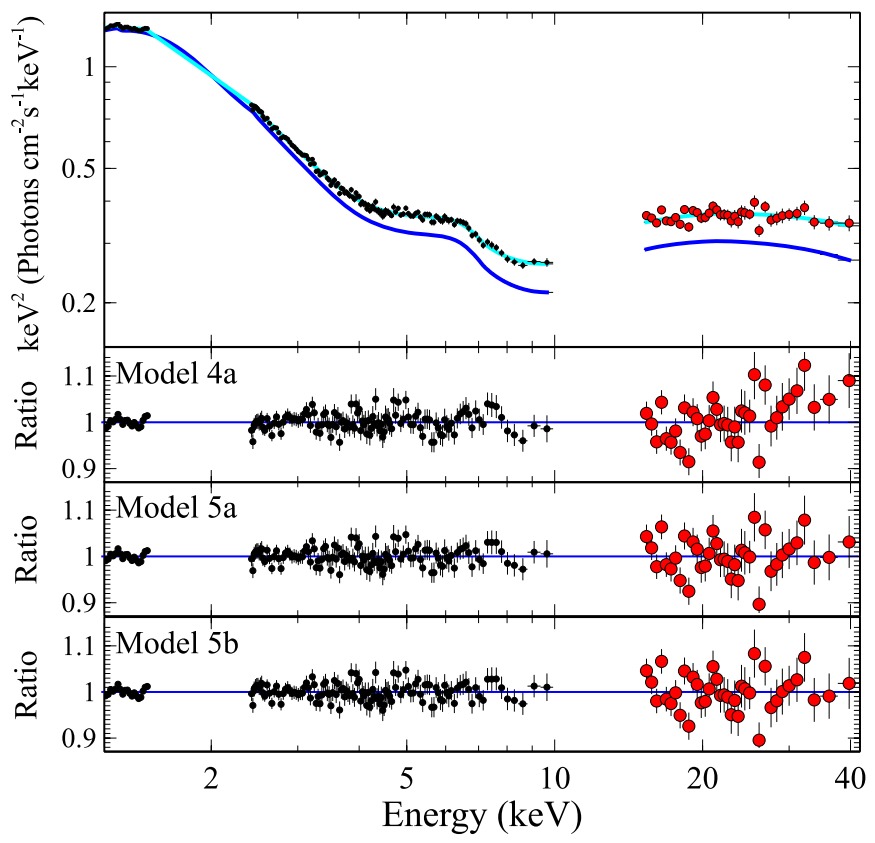

Figure 5. From top to bottom: response unfolded $v F v$ spectrum to the best-fit Model 5b. The total and blurred REFBHB components are shown as cyan and blue solid lines, respectively. The power-law continuum falls below the $y$-scale; data/model ratio for: (4a) phabs $*($ diskbb + powerlaw $+k d$ blur $\otimes$ reflion $)$; (5a) phabs $*$ (powerlaw + kdblur $\otimes$ ref bhb); and (5b) phabs $*($ powerlaw + relconv $\otimes$ refbhb).

(A color version of this figure is available in the online journal.)

with disk emission (DISKBB) and a power-law illuminating continuum. The photon indices of the REFLIONX and powerlaw components are assumed to be the same. The outer disk radius is assumed to be at $400 r_{\mathrm{g}}$ (the maximum allowed by the model) and the iron abundance of MAXI J1836-194 is fixed at solar. This model gives a poor fit with $\chi^{2} / v=400.6 / 324$. Allowing instead for a broken power-law emissivity profile results in a significant improvement with $\chi^{2} / v=379.7 / 322$. This model is described in detail in Table 2 (Model 4a) and shown in Figure 5.

It is worth noting some similarities and differences between the values obtained from the current reflection model and those from the phenomenological fits described in the previous section. To start, the high ionization parameter found here 
Table 2

Fits with Physically Motivated Reflection Models

\begin{tabular}{lcccc}
\hline \hline & Model 4a & Model 4b & Model 5a & Model 5b \\
\hline$N_{\mathrm{H}}\left(\times 10^{22} \mathrm{~cm}^{-2}\right)$ & $0.11 \pm 0.02$ & $0.11(\mathrm{f})$ & $0.191_{-0.030}^{+0.003}$ & $0.19 \pm 0.03$ \\
$\Gamma$ & $1.92 \pm 0.04$ & $1.92(\mathrm{f})$ & $1.82_{-0.01}^{+0.06}$ & $1.81_{-0.03}^{+0.05}$ \\
$k T_{\text {disk }}(\mathrm{keV})$ & $0.448_{-0.008}^{+0.006}$ & $0.448(\mathrm{f})$ & $0.27_{-0.01}^{+0.02}$ & $0.26_{-0.03}^{+0.02}$ \\
$N_{\text {hard }}$ & $<0.01$ & $<0.01$ & $0.041_{-0.002}^{+0.014}$ & $0.030_{-0.004}^{+0.077}$ \\
$N_{\text {diskbb }}\left(\times 10^{3}\right)$ & $4.5_{-0.4}^{+0.5}$ & $4.5(\mathrm{f})$ & $\ldots$ & $\ldots$ \\
$F_{\text {Illum }} / F_{\mathrm{BB}}$ & $\ldots$ & $\ldots$ & $1.2 \pm 0.2$ & $1.2_{-0.3}^{+0.2}$ \\
$n_{\mathrm{H}}\left(\times 10^{19}\right)$ & $\ldots$ & $\ldots$ & $2.5_{-1.0}^{+0.7}$ & $2.9_{-0.6}^{+1.7}$ \\
$N_{\text {refbhb }}$ & $\ldots$ & $\ldots$ & $0.31_{-0.04}^{+0.03}$ & $0.30_{-0.04}^{+0.02}$ \\
$N_{\text {reflionx }}\left(\times 10^{-6}\right)$ & $3.7 \pm 0.4$ & $4.5_{-0.6}^{+0.5}$ & $\ldots$ & $\ldots$ \\
$q_{\text {in }}$ & $>5.8$ & $>4.4$ & $>6.5$ & $>7.3$ \\
$q_{\text {out }}$ & $3.4 \pm 0.1$ & $3.7 \pm 0.1$ & $3.12_{-0.12}^{+0.06}$ & $3.19_{-0.05}^{+0.07}$ \\
$r_{\text {break }}\left(r_{\mathrm{g}}\right)$ & $3.6_{-0.2}^{+2.3}$ & $3.2_{-0.1}^{+2.1}$ & $3.4_{-0.1}^{+0.2}$ & $3.6_{-0.1}^{+0.2}$ \\
$\theta(\mathrm{deg})$ & $<15$ & $<30$ & $<23$ & $<17$ \\
$\xi$ & $2150_{-250}^{+170}$ & $2170_{-250}^{+500}$ & $\ldots$ & $\ldots$ \\
$r_{\text {in }}\left(r_{\mathrm{g}}\right)$ & $2.54_{-0.20}^{+0.06}$ & $1.8_{-0.3}^{+0.8}$ & $2.2_{-0.4}^{+0.2}$ & $\left(2.45_{-0.20}^{+0.18}\right)^{\mathrm{a}}$ \\
Spin $(a)$ & $\ldots$ & $\ldots$ & $\ldots$ & $0.88 \pm 0.03$ \\
$\chi^{2} / v$ & $379.7 / 322$ & $198.5 / 211$ & $344.9 / 322$ & $344.5 / 322$ \\
\hline & & & &
\end{tabular}

Notes. Model 4 is described in XSPEC as phabs $*($ diskbb + powerlaw + $k d$ blur $*$ reflion $x$ ). Model $4 \mathrm{~b}$ is identical to $4 \mathrm{a}$ but we only fit the $3-10 \mathrm{keV}$ energy range. Model 5 replaces REFLIONX and DISKBB with the fully selfconsistent reflection model REFBHB. In all models described so far the kernel from the LAOR line profile was used to account for the gravitational effects close to the black hole. In Model 5b, we finally replaces the KDBLUR kernel with the relativistic code RELCONV were the spin is a parameter of the model. In all cases the hard emission illuminating the disk is assumed to be a power law with index $\Gamma$. All errors are $90 \%$ confidence for one parameter.

${ }^{a}$ Inner radius is not a model parameter and was derived by using the relationship between the spin and ISCO (Bardeen et al. 1972). It is shown here merely to allow for easy comparison.

$\left(\xi \approx 2200 \mathrm{erg} \mathrm{cm} \mathrm{s}^{-1}\right)$ is similar to that found in the most physically motivated version of Model 3 (i.e., Model 3d) and suggests an intermediate- to high-ionization state. The moderate-to-low disk inclination shown in Figure 4 is also confirmed here, where the current reflection fit suggests $\theta \lesssim$ $15^{\circ}$. The disk parameters found here are also in close agreement with those found in Model 2, however we cannot compare the parameters with Model 3 as the disk was modeled assuming a Comptonization model. A major difference between the fit with the self-consistent reflection component over that of the various phenomenological models is the necessity for a broken emissivity profile over that of a single power law. In Model 4a, the disk extends to within $\sim 2.5 r_{\mathrm{g}}$. The steep emissivity occurs in a very narrow annuli between this radius and $\sim 4 r_{\mathrm{g}}$, at which point it goes back to the value expected from a purely Newtonian geometry. It is interesting to note that in the phenomenological Model 3d, the inner radius obtained with an emissivity index of $\sim 3.3$ is similar to the break radius found here. In the case of the phenomenological models, the inner radius was obtained from a pure emission line. The approach of using a model such as the LAOR line profile to obtain spin has many limitations as the information imprinted by the effects of strong gravity is not limited to an emission line. In fact, such a limitation is clear in the inconsistency between the high ionization found here $\left(\xi \sim 2200 \mathrm{erg} \mathrm{cm} \mathrm{s}^{-1}\right)$ as well as that found in Model $3 \mathrm{~d}\left(\xi \sim 5000 \mathrm{erg} \mathrm{cm} \mathrm{s}^{-1}\right)$ with the line centroid of $\sim 6.48 \mathrm{keV}$ found in that same model which indicates lowly ionized iron. In

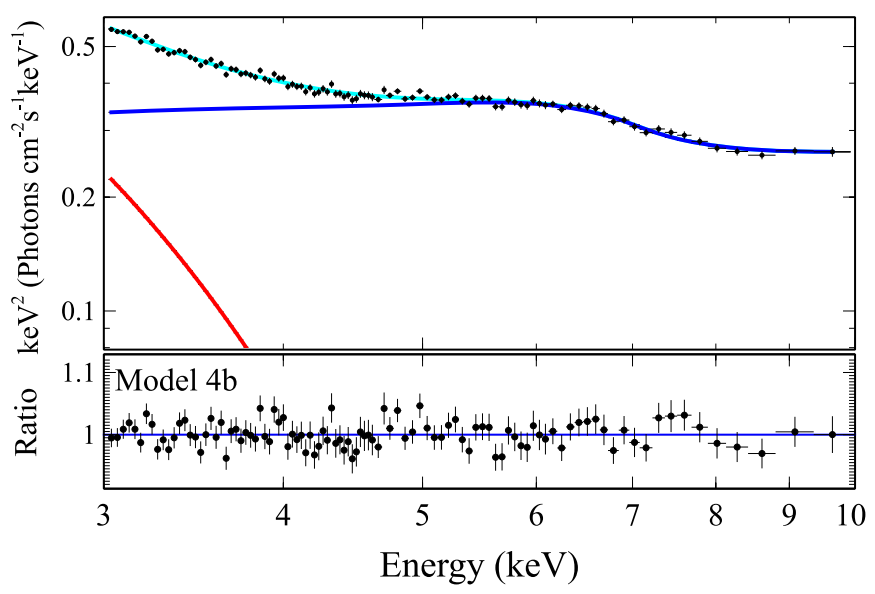

Figure 6. Response unfolded $v F v$ spectrum from Model $4 \mathrm{~b}$ fit in the $3-10 \mathrm{keV}$ energy range. The total, blurred reflection and disk components are shown as cyan, blue, and red solid lines, respectively. The power-law continuum falls below the $y$-scale. Bottom: data model ratio to Model 4b. The reflection parameters obtained in this range are identical to that obtained by modeling the full spectra (see Table 2).

(A color version of this figure is available in the online journal.)

Model 3d, a further constraint on the inner radius was in place by the act of convolving the PEXRIV model with the same kernel as the LAOR line profile. Here, there was an interplay between broadening of the emission line component as well as the iron absorption edge (modeled separately and therefore not forced to be physical). The final product was the apparent presence of an emission line at $\sim 6.48 \mathrm{keV}$, as expected from cold matter with $\xi \lesssim 100 \mathrm{erg} \mathrm{cm} \mathrm{s}^{-1}$, together with the absorption edge from hot hydrogenic iron Ka Fe XXV and/or Fe XXVI with edge energies $\gtrsim 8.85 \mathrm{keV}$ as expected from $500 \lesssim \xi \lesssim 5000 \mathrm{erg} \mathrm{cm} \mathrm{s}^{-1}$. This combination acted to model a broad feature with only a mild degree of relativistic broadening. REFLIONX on the other hand, by virtue of the imposed self-consistency in its atomic physics, modeled the same broad feature by allowing a greater degree of broadening to be attributed to gravity, and hence resulted in a smaller inner radius.

A question that generally arises when one is dealing with reflection models is whether the constraints on the various parameters of interest (i.e., inner radius/spin, disk inclination, and emissivity profile, etc) are driven by anything other than the reflection features. For example, could it be that extreme blurring, which would suggest a maximally rotating black hole, is artificially caused by the model trying to "smooth" the soft part of the reflection spectrum to mimic a disk component? To test this we ignored the XIS data below $3 \mathrm{keV}$ where the disk emission dominates (see Figure 2) and removed the PIN data altogether. What remained is essentially the broad feature which we are associating with iron fluorescence emission. We refit Model 4a after freezing the disk temperature and its normalization as well the neutral hydrogen column density and power-law index, as these cannot be constrained from the line profile alone. We refer to this as Model $4 \mathrm{~b}$ in Table 2, and show this fit in Figure 6. It is clear that all parameters obtained from this narrow energy range are consistent, within errors, to that obtained using the full spectra. Allowing $\Gamma$ to vary does not change this conclusion.

Until this point, we have made use of REFLIONX, which is designed to reproduce reflection spectra from the accretion disks around AGNs. However, the disks around stellar mass black holes are significantly hotter, resulting in subtle differences 

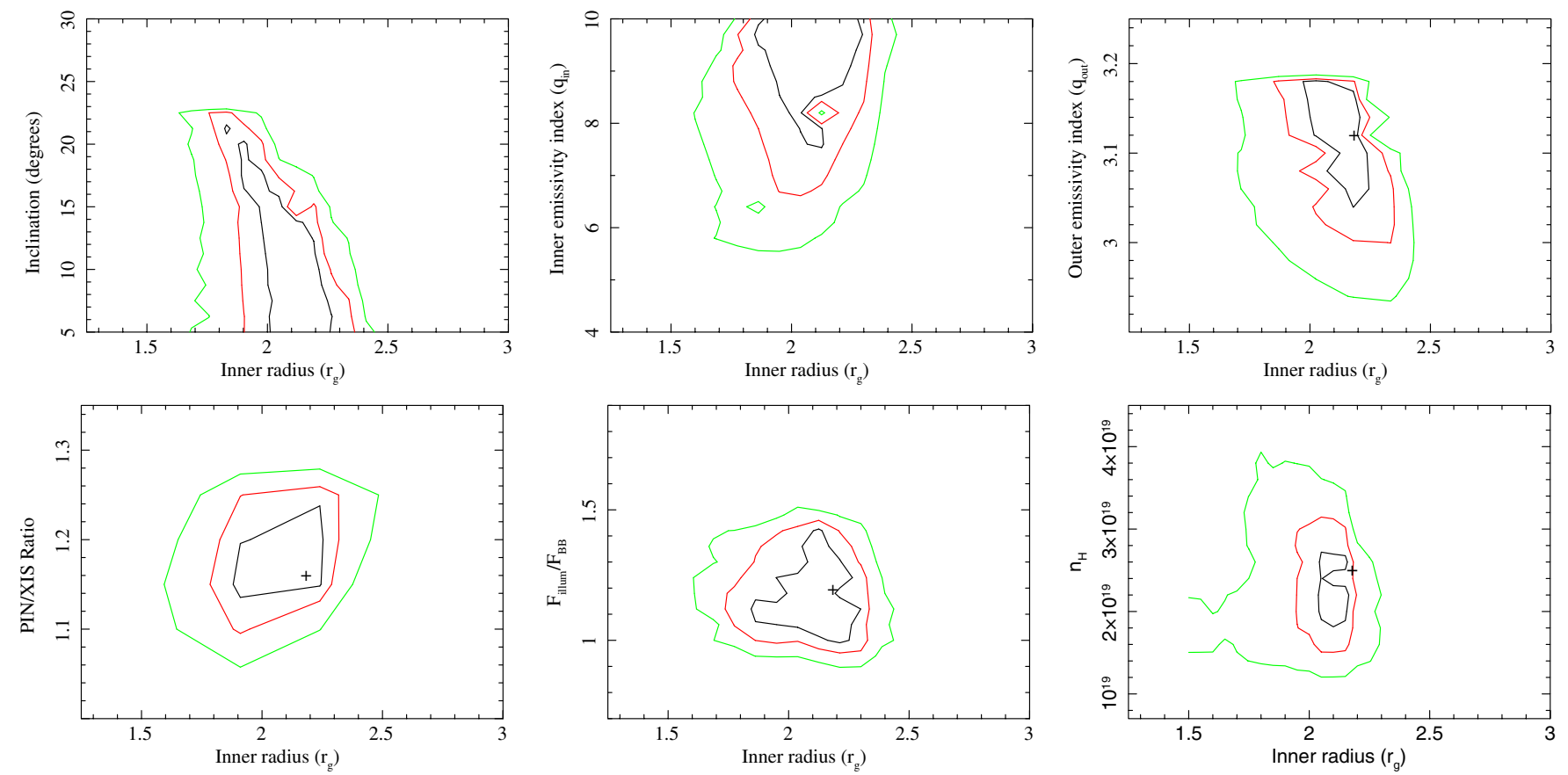

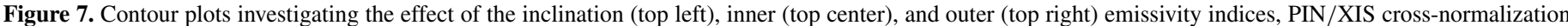

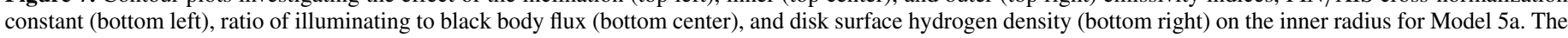

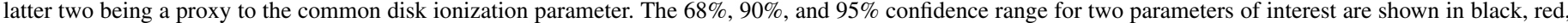

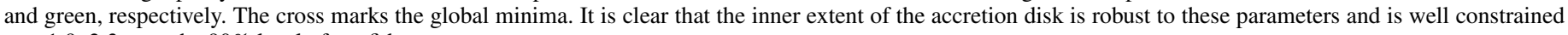
to $\sim 1.9-2.3 r_{\mathrm{g}}$ at the $90 \%$ level of confidence.

(A color version of this figure is available in the online journal.)

in the radiation processes, including reprocessing. The higher disk temperatures around stellar mass black holes mean that Compton broadening is of greater importance and should therefore be included at the correct level. With this in mind, Ross $\&$ Fabian (2007) developed a modified version of the previous grid, REFBHB, in which the atmosphere of the accretion disk is illuminated not only by the hard, power-law-like corona, but also by a further blackbody radiation intrinsic to the disk. This model self-consistently accounts for the disk, relativistic line, and reflection continuum present in the phenomenological models described in the previous section.

The parameters of the model are the number density of hydrogen in the illuminated atmosphere, $n_{\mathrm{H}}$, the temperature of the accretion disk, $k T_{\text {disk }}$ the index of the (assumed power law) continuum, and the ratio of the total flux illuminating the disk to the total blackbody flux emitted by the disk, $F_{\text {illum }} / F_{\mathrm{BB}}$. The disk reflection spectra are again convolved with KDBLUR to account for relativistic effects. This model (Model 5a; Table 2 and shown in Figure 5) provides the best fit yet to the data with $\chi^{2} / v=$ $344.9 / 322$ despite being more constrained - compared to purely phenomenological models - by virtue of being physically selfconsistent. The inner accretion disk radius of $r_{\text {in }}=2.2_{-0.4}^{+0.2} r_{\mathrm{g}}$ is consistent with the values found from the REFLIONX model together with a separate disk component. Assuming that this radius is the same as the radius of the innermost stable circular orbit, we can constrain the spin parameter (Bardeen et al. 1972) to be $a=0.915_{-0.03}^{+0.05}$.

We again investigate the dependence of the inner radius $r_{\text {in }}$ on a number of key parameters including the inclination angle, the inner and outer emissivity indices, the cross-normalization between the XIS and PIN data, the ratio of the illuminating power law to the blackbody flux, and the hydrogen number density at the disk surface. Figure 7 shows that the inner radius found here of $r_{\text {in }}=2.2_{-0.4}^{+0.2} r_{\mathrm{g}}$ is extremely robust to changes in all the aforementioned parameters. However, in order to make a formal constraint on the spin, we replace KDBLUR with the sophisticated variable-spin relativistic smearing model RELCONV (Dauser et al. 2010). Figure 8 shows that the spin of the black hole in MAXI J1836-194 is well constrained to be $a=0.88 \pm 0.03$ at the $90 \%$ confidence range. In the following, section we will discuss, among other things, the current strength and limitation of the various models used throughout this work and highlight some of the factors contributing to the tight constraint on the spin parameter of MAXI J1836-194.

\section{DISCUSSION}

The fractional contribution of the disk component to the total $2-20 \mathrm{keV}$ flux $(\sim 25 \%)$ places the current observation in the hard/intermediate state as defined by Belloni (2010) and in an intermediate state between the low/hard and high/ soft state definition of McClintock et al. (2006). However, we can see from Figures 5 and 6 that the observed continuum is not dominated by the power-law-like component expected to originate from the corona, but rather it is mostly reflection dominated. Similar "reflection-dominated" spectra are seen in a number of narrow line Seyfert 1 galaxies and quasars during their low state (e.g., 1H0419-577, Fabian et al. 2005; NGC 4051, Ponti et al. 2006; PG1543+489, Vignali et al. 2008; Mrk 335, Grupe et al. 2008; PG1535+547, Ballo et al. 2008; PG2112+059, Schartel et al. 2010). In this scenario, the majority of the X-ray reprocessing (reflection) occurs in the inner region of the accretion disk where strong gravitational light bending is expected to occur (Martocchia \& Matt 1996; Miniutti \& Fabian 2004). Such behavior is expected as a result of strong light bending, where the reflected flux is enhanced 


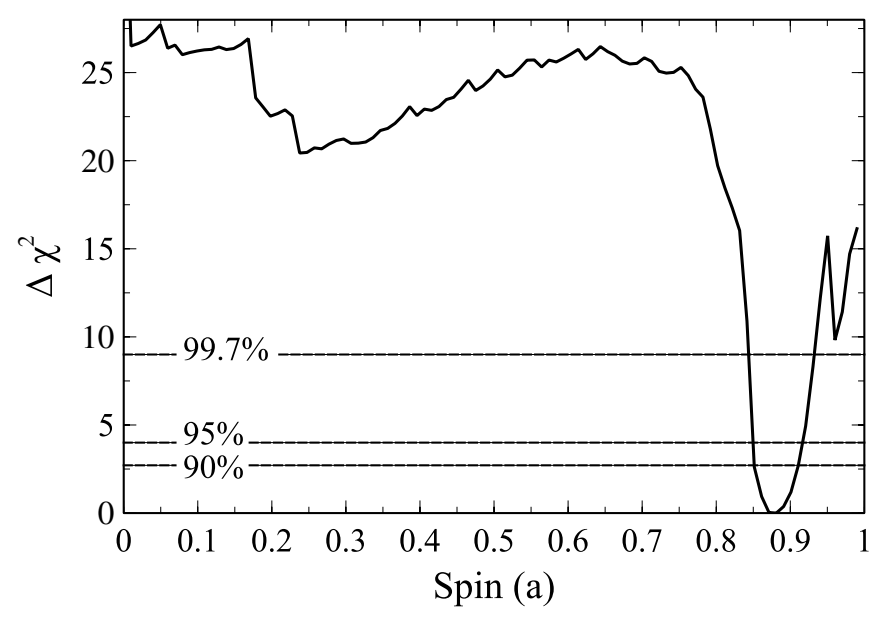

Figure 8. Goodness of fit vs. spin for Model 5b. It is clear that the black hole in MAXI J1836-194 is rapidly rotating. A maximally rotating Kerr or a static Schwarzschild black hole are both rejected at greater than the $3 \sigma$ level of confidence.

over the inner regions as a result of gravitational focusing of the $\mathrm{X}$-ray continuum down toward the black hole and onto the disk. The decrease in the number of X-rays that can escape as part of the continuum thus causes the source to appear reflection dominated.

Wilkins \& Fabian (2011) showed that a possible consequence of strong gravitation effects is an increase in the emissivity profile of the disk. Classically, the emissivity profile is expected to be flat in the region directly below the source, while tending to $r^{-3}$ when $r \gg h$, where the flux received by the disk from the source falls off as the inverse square of the distance with a further factor of $1 / r$ arising from the cosine of the angle projecting the ray normal to the disk plane. However, as briefly mentioned above and detailed in Wilkins \& Fabian (2011), strong gravity can potentially act to focus more of the direct continuum into the inner parts of the disk as well as increase the disk area being radiated-the latter as a consequence of gravitational warping. In this particular treatment, these factors cause for a substantial steepening of the emissivity profile in the inner regions.

This is indeed a possible explanation for what is observed in MAXI J1836-194, where within a radius of $\lesssim 4 r_{\mathrm{g}}$ the emissivity index is consistently $>6$ (see Table 2) and beyond it goes closer to the classical value of 3 . Both the reflection-dominated spectrum and the high emissivity profile seen here suggest that the primary X-ray continuum is located within a few gravitational radii of the black hole. Looking at Figure 1, it is indeed possible that the corona briefly "collapsed" down close to the black hole, causing the decrease in the hard X-ray flux seen during this Suzaku observation. If this is the case, state transitions (at least that between the high/soft and low/hard) should be seen to be much more tightly associated with changes in the corona as opposed to physical changes in the accretion disk.

In this work, we have investigated a variety of possible models striving toward a physically motivated interpretation for the observed spectrum. It is worth stressing that equally good fits - and at times (albeit not in this work) statistically better fits - can be obtained with a purely phenomenological combination of components severely lacking in physical consistency. A case in point is Model 3c where the presence of the-clearly broad-iron line requires the emission to occur from deep within $6 r_{\mathrm{g}}$, yet the direct X-ray and reflection continuum appear some-

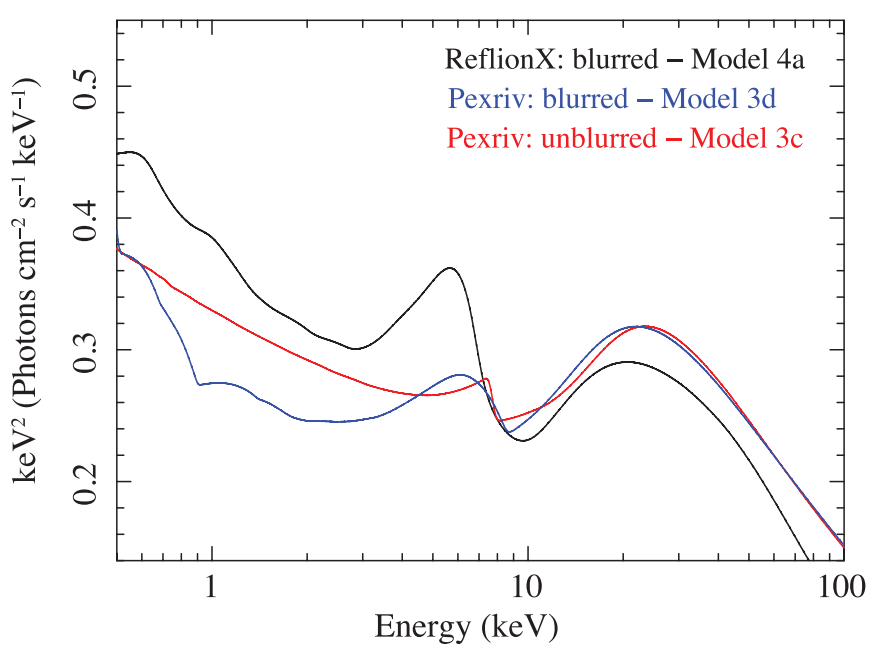

Figure 9. Difference between the blurred REFLIONX component in Model 4a (black) to the PEXRIV components in Models 3d (blue) and 3c (red). The unblurred PEXRIV (red) is as expected from a "cold" $\left(\xi=70 \mathrm{erg} \mathrm{cm} \mathrm{s}^{-1}\right)$ accretion disk being illuminated by a $\Gamma=2.18$ power law without being relativistic convolved. Compare this to the component expected from a moderately ionized $\left(\xi=5200 \mathrm{erg} \mathrm{cm} \mathrm{s}^{-1}\right.$; blue) disk at $3.3 r_{\mathrm{g}}$ illuminated by a $\Gamma=2.09$ power law and the REFLIONX model having both the absorption edge and the iron emission line (black).

(A color version of this figure is available in the online journal.)

how exempt from the effect of strong gravity. When we account for relativistic effects, the ionization parameter of the disk increases by nearly two orders of magnitude from $\xi \approx 70$ to $\approx 5000 \mathrm{erg} \mathrm{cm} \mathrm{s}^{-1}$. This change is accompanied by a hardening of the photon index $\Gamma$ possibly due to the power law trying to compensate for the stronger soft emission and the weaker Compton hump of the blurred ionized reflector in comparison to the unblurred, cold reflector (see Figure 9).

A further point to note is that the apparent decrease in the equivalent width of the relativistic line profile (from $270 \mathrm{eV}$ to $180 \mathrm{eV}$ from Model 3c to Model 3d) is clearly a consequence of the, physically inconsistent, lack of gravitational blurring. From Figure 9 we can see that the absorption edge in PEXRIV becomes much more smooth and symmetric after the component is convolved with KDBLUR and the ionization increases. Due to the decoupling between the LAOR line profile and PEXRIV, this smooth edge can act somewhat like an emission line conspiring against the LAOR line component and thus decreasing the equivalent width of latter. In order to couple the emission line strength with the absorption edge and all other reflection features, we replaced the phenomenological combination of LAOR + PEXRIV with the REFLIONX reflection grid. The first thing to note from Table 2 (model 4) is that this recovered the high-ionization value from Model $3 \mathrm{~d}$ but now in a selfconsistent manner. Nonetheless, the strong requirement for an emission line with an equivalent width of at least $\approx 180 \mathrm{eV}$ (Model 3d) is highly indicative of a broad line, adhering to the strong criterion of Reis et al. (2010) arguing against an accretion disk truncated far from the ISCO.

The precise value of the neutral hydrogen column density toward MAXI J1836-194 is not known; however, based on Swift/XRT data which extend to much lower energies compared to the current Suzaku observation, Kennea et al. (2011) showed that a likely value is $(2 \pm 0.4) \times 10^{21} \mathrm{~cm}^{-2}$. A similar value is found here using our best model. Figure 9 highlights how differing models impact the energies below $\sim 1 \mathrm{keV}$ where we would begin to see the curvatures expected from such a low 
column density. The fact that our data cut off at $1.2 \mathrm{keV}$ means that, in order to break the degeneracies between the-essentially disk-continuum (see Figure 2) and neutral column density, one must use a self-consistent model for the full spectra. Such is the case for REFBHB.

It is well known that in the hot inner regions of an accretion disk the observed disk spectrum suffers from the effects of electron scattering, which results in an observed (color) temperature, $T_{\mathrm{col}}$, that is higher than the effective blackbody temperature, $T_{\mathrm{bb}}$, by approximately a factor of $f_{\mathrm{col}}=T_{\mathrm{col}} / T_{\mathrm{bb}}$ (Ross et al. 1992). This "color correction factor" has been shown to have a value of $1.7 \pm 0.2$ (Shimura \& Takahara 1995) for a wide range in luminosity, as long as the disk effective temperature remains below $\sim 1 \mathrm{keV}$ (Davis et al. 2005), as is the case here. Above this temperature, disk self-ionization can lead to an increase in $f_{\text {col }}$ however it is found to be consistently below $\sim 3$ (Merloni et al. 2000). The effective temperature in the REFBHB (Model 5) of $k T_{5}=0.27_{-0.01}^{+0.02} \mathrm{keV}$ is precisely as expected from the value of the color temperature from the DISKBB component in Model 4 $\left(k T_{4} / k T_{5}=1.7 \pm 0.1\right)$.

Last, although we cannot directly compare $\xi$ between REFLIONX and REFBHB, we can estimate this value based on the various parameters output in Table 2 . Since the flux of the blackbody in the model is related to its temperature by the $F_{\mathrm{bb}} \propto T^{4}$ relation, we find $F_{\mathrm{bb}}=\left(5.5_{-0.8}^{+1.8} \times 10^{21} \mathrm{erg} \mathrm{cm}^{-2} \mathrm{~s}^{-1}\right.$. Combining this $F_{\text {Illum }} / F_{\mathrm{BB}}=1.2 \pm 0.2$ gives an illuminating flux of $\left(6.5_{-1.4}^{+2.4}\right) \times 10^{21} \mathrm{erg} \mathrm{cm}^{-2} \mathrm{~s}^{-1}$. The ionization parameter, which is defined as $\xi=4 \pi F / n \mathrm{erg} \mathrm{cm} \mathrm{s}^{-1}$, is then found by using $n=n_{\mathrm{H}}=2.5_{-1.0}^{+0.7} \times 10^{19} \mathrm{Hcm}^{-3}$. In this manner, we find $\xi=3300 \pm 1500 \mathrm{erg} \mathrm{cm} \mathrm{s}^{-1}$, in perfect agreement with the values found for both REFLIONX in both Models $4 \mathrm{a}$ and $4 \mathrm{~b}$ and the blurred PEXRIV in Model 3d.

All current methods of measuring black hole spin rely on both the assumption that the accretion disk extends to the innermost stable circular orbit and that emission within this radius is negligible. The latter is indeed valid for the standard model of black hole accretion (Shakura \& Sunyaev 1973), where within the ISCO_-in the region often referred to as the plunging region - there is no angular momentum transport, and the region cannot support an X-ray corona to irradiate the material that is ballistically plunging onto the black hole. However, it was shown by Krolik (1999) and independently by Gammie (1999) that when magnetic fields are considered, the B-fields within the ISCO may be amplified to a point where the magnetic energy density can be comparable to the rest-mass energy of the accreting material and may lead to the creation of an active inner X-ray corona. Shafee et al. (2008) investigated the effect of magnetic torque within the plunging region around a nonrotating black hole and concluded that "...magnetic coupling across the ISCO is relatively unimportant for geometrically thin disks."

A further study specifically aimed at addressing the robustness of the iron line/reflection fitting technique in diagnosing black hole spin was presented by Reynolds \& Fabian (2008). The authors used a high-resolution three-dimensional magnetohydrodynamic simulation of a geometrically thin accretion disk to show that the density of the plunging material drops precipitously over a very small radius within the ISCO. This sudden drop in density results in the material being highly photoionized and suppresses any significant iron line emission as well as all other reflection features from within the ISCO. The study by Reynolds \& Fabian (2008) concluded that for a non-rotating black hole where the ISCO is at $6 r_{\mathrm{g}}$, the reflection edge-defined by the authors as the innermost radius from which significant reflection emission is seen-is at approximately $5.8 r_{\mathrm{g}}$. Furthermore, the discrepancy between the true ISCO and the inferred radius diminishes as one considers more rapidly rotating black holes, as is the case in MAXI J1836-194 (see Figure 8). From an observational perspective, it is also worth noting that strong support for the presence of an "inner edge" in the accretion disk surrounding black holes is provided by decades of empirical evidence as shown in Steiner et al. (2010).

The culmination of this work is that the recently discovered system, MAXI J1836-194, is indeed a stellar mass black hole binary, having a central black hole rotating with a spin parameter of $a=0.88 \pm 0.03$ (90\% confidence). This strong constraint is a result of being able to successfully, and most importantly self-consistently, model the reflection features clearly present in the Suzaku spectra.

R.C.R. thanks the Michigan Society of Fellows and NASA; the Suzaku team, which scheduled this TOO observation; and the MAXI team for providing prompt notice of this new source to the astronomical community at large. R.C.R. is supported by NASA through the Einstein Fellowship Program, grant No. PF1-120087 and is a member of the Michigan Society of Fellows. A.C.F. thanks the Royal Society. This work was greatly expedited thanks to the help of Jeremy Sanders in optimizing the various convolution models.

\section{REFERENCES}

Ballantyne, D. R., Ross, R. R., \& Fabian, A. C. 2001, MNRAS, 327, 10

Ballo, L., Giustini, M., Schartel, N., et al. 2008, A\&A, 483, 137

Bardeen, J. M., Press, W. H., \& Teukolsky, S. A. 1972, ApJ, 178, 347

Belloni, T. M. (ed.) 2010, in States and Transitions in Black Hole Binaries

(Lecture Notes in Physics, Vol. 794; Berlin: Springer), 53

Bhattacharyya, S., \& Strohmayer, T. E. 2007, ApJ, 664, L103

Blum, J. L., Miller, J. M., Fabian, A. C., et al. 2009, ApJ, 706, 60

Boldt, E. 1987, in IAU Symp. 124, Observational Cosmology, ed. A. Hewitt,

G. Burbidge, \& L. Z. Fang (Cambridge: Cambridge Univ. Press), 611

Brenneman, L. W., Reynolds, C. S., Nowak, M. A., et al. 2011, ApJ, 736, 103

Cackett, E. M., Altamirano, D., Patruno, A., et al. 2009, ApJ, 694, L21

Cackett, E. M., Miller, J. M., Ballantyne, D. R., et al. 2010, ApJ, 720, 205

Cackett, E. M., Miller, J. M., Bhattacharyya, S., et al. 2008, ApJ, 674, 415

Cenko, S. B., Miller, A. A., \& Bloom, J. S. 2011, ATel, 3614, 1

Dauser, T., Wilms, J., Reynolds, C. S., \& Brenneman, L. W. 2010, MNRAS, 409, 1534

Davis, J. E. 2001, ApJ, 562, 575

Davis, S. W., Blaes, O. M., Hubeny, I., \& Turner, N. J. 2005, ApJ, 621, 372

Del Santo, M., Kuulkers, E., Rodriguez, J., et al. 2011, ATel, 3652, 1

di Salvo, T., D’Aí, A., Iaria, R., et al. 2009, MNRAS, 398, 2022

Done, C., \& Diaz Trigo, M. 2010, MNRAS, 407, 2287

Done, C., Gierliński, M., \& Kubota, A. 2007, A\&AR, 15, 1

Ebisawa, K., Ogawa, M., Aoki, T., et al. 1994, PASJ, 46, 375

Esin, A. A., McClintock, J. E., \& Narayan, R. 1997, ApJ, 489, 865

Fabian, A. C., Miniutti, G., Iwasawa, K., \& Ross, R. R. 2005, MNRAS, 361, 795

Fabian, A. C., Zoghbi, A., Ross, R. R., et al. 2009, Nature, 459, 540

Fabian, A. C., et al. 2012, MNRAS, submitted

Fender, R. P., Gallo, E., \& Russell, D. 2010, MNRAS, 406, 1425

Gammie, C. F. 1999, ApJ, 522, L57

García, J., \& Kallman, T. R. 2010, ApJ, 718, 695

García, J., Kallman, T. R., \& Mushotzky, R. F. 2011, ApJ, 731, 131

George, I. M., \& Fabian, A. C. 1991, MNRAS, 249, 352

Gou, L., McClintock, J. E., Liu, J., et al. 2009, ApJ, 701, 1076

Grupe, D., Komossa, S., Gallo, L. C., et al. 2008, ApJ, 681, 982

Hiemstra, B., Méndez, M., Done, C., et al. 2011, MNRAS, 411, 137

Kennea, J. A., Hoversten, E. A., Siegel, M. H., et al. 2011, ATel, 3613, 1

Kolehmainen, M., Done, C., \& Díaz Trigo, M. 2011, MNRAS, 416, 311

Koyama, K., Tsunemi, H., Dotani, T., et al. 2007, PASJ, 59, 23

Krolik, J. H. 1999, ApJ, 515, L73

Laor, A. 1991, ApJ, 376, 90

Lightman, A. P., \& White, T. R. 1988, ApJ, 335, 57 
Magdziarz, P., \& Zdziarski, A. A. 1995, MNRAS, 273, 837

Martocchia, A., \& Matt, G. 1996, MNRAS, 282, L53

Matt, G., Perola, G. C., \& Piro, L. 1991, A\&A, 247, 25

McClintock, J. E., Shafee, R., Narayan, R., et al. 2006, ApJ, 652, 518

Merloni, A., Fabian, A. C., \& Ross, R. R. 2000, MNRAS, 313, 193

Miller, J. M. 2007, ARA\&A, 45, 441

Miller, J. M., D’Aì, A., Bautz, M. W., et al. 2010, ApJ, 724, 1441

Miller, J. M., Homan, J., Steeghs, D., et al. 2006, ApJ, 653, 525

Miller, J. M., Miller, M. C., \& Reynolds, C. S. 2011, ApJ, 731, L5

Miller, J. M., Reynolds, C. S., Fabian, A. C., Miniutti, G., \& Gallo, L. C. 2009, ApJ, 697, 900

Miller, J. M., Reynolds, C. S., Fabian, A. C., et al. 2008, ApJ, 679, L113

Miller-Jones, J. C. A., Sivakoff, G. R., Rupen, M., \& Altamirano, D. 2011, ATel, 3628,1

Miniutti, G., \& Fabian, A. C. 2004, MNRAS, 349, 1435

Miniutti, G., Panessa, F., de Rosa, A., et al. 2009, MNRAS, 398, 255

Mitsuda, K., Inoue, H., Koyama, K., et al. 1984, PASJ, 36, 741

Nakahira, S., Koyama, S., Ueda, Y., et al. 2012, PASS, 64, 13

Nakahira, S., Negoro, H., Usui, R., et al. 2011, ATel, 3626, 1

Nardini, E., Fabian, A. C., Reis, R. C., \& Walton, D. J. 2011, MNRAS, 410, 1251

Nayakshin, S., Kazanas, D., \& Kallman, T. R. 2000, ApJ, 537, 833

Negoro, H., Nakajima, M., Nakahira, S., et al. 2011, ATel, 3611, 1

Ponti, G., Miniutti, G., Cappi, M., et al. 2006, MNRAS, 368, 903

Poutanen, J., \& Svensson, R. 1996, ApJ, 470, 249

Rau, A., Greiner, J., \& Sudilovsky, V. 2011, ATel, 3619, 1

Reis, R. C., Fabian, A. C., \& Miller, J. M. 2010, MNRAS, 402, 836

Reis, R. C., Fabian, A. C., Reynolds, C. S., et al. 2012, ApJ, 745, 93

Reis, R. C., Fabian, A. C., Ross, R. R., \& Miller, J. M. 2009a, MNRAS, 395, 1257

Reis, R. C., Fabian, A. C., Ross, R. R., et al. 2008, MNRAS, 387, 1489
Reis, R. C., Fabian, A. C., \& Young, A. J. 2009b, MNRAS, 399, L1

Reis, R. C., Miller, J. M., Fabian, A. C., et al. 2011, MNRAS, 410, 2497

Remillard, R. A., \& McClintock, J. E. 2006, ARA\&A, 44, 49

Reynolds, C. S., \& Fabian, A. C. 2008, ApJ, 675, 1048

Ross, R. R., \& Fabian, A. C. 1993, MNRAS, 261, 74

Ross, R. R., \& Fabian, A. C. 2005, MNRAS, 358, 211

Ross, R. R., \& Fabian, A. C. 2007, MNRAS, 381, 1697

Ross, R. R., Fabian, A. C., \& Mineshige, S. 1992, MNRAS, 258, 189

Russell, D. M., Belloni, T., Miller-Jones, J. C. A., et al. 2011, ATel, 3689, 1

Schartel, N., Rodríguez-Pascual, P. M., Santos-Lleó, M., et al. 2010, A\&A, 512, A75

Schmoll, S., Miller, J. M., Volonteri, M., et al. 2009, ApJ, 703, 2171

Shafee, R., McKinney, J. C., Narayan, R., et al. 2008, ApJ, 687, L25

Shakura, N. I., \& Sunyaev, R. A. 1973, A\&A, 24, 337

Shimura, T., \& Takahara, F. 1995, ApJ, 445, 780

Steiner, J. F., McClintock, J. E., Remillard, R. A., et al. 2010, ApJ, 718, L117

Steiner, J. F., Reis, R. C., McClintock, J. E., et al. 2011, MNRAS, 416, 941

Strohmayer, T. E., \& Smith, E. A. 2011, ATel, 3618, 1

Takahashi, T., Abe, K., Endo, M., et al. 2007, PASJ, 59, 35

Tanaka, Y., Nandra, K., Fabian, A. C., et al. 1995, Nature, 375, 659

Tomsick, J. A., Yamaoka, K., Corbel, S., et al. 2009, ApJ, 707, L87

Trushkin, S. A., Nizhelskij, N. A., \& Zhekanis, G. V. 2011, ATel, 3656, 1

Uchiyama, Y., Maeda, Y., Ebara, M., et al. 2008, PASJ, 60, 35

Vignali, C., Piconcelli, E., Bianchi, S., \& Miniutti, G. 2008, MNRAS, 388, 761

Walton, D. J., Reis, R. C., Cackett, E. M., Fabian, A. C., \& Miller, J. M. 2012, arXiv: 1202.5193

Walton, D. J., Reis, R. C., \& Fabian, A. C. 2010, MNRAS, 408, 601

Wilkins, D. R., \& Fabian, A. C. 2011, MNRAS, 414, 1269

Wilkinson, T., \& Uttley, P. 2009, MNRAS, 397, 666

Zycki, P. T., Krolik, J. H., Zdziarski, A. A., \& Kallman, T. R. 1994, ApJ, 437, 597 